\title{
Refining drug administration in a murine model of acute infection with Trypanosoma cruzi
}

\author{
Julián Ernesto Nicolás Gulin ${ }^{1,2^{*}}$ (D), Margarita Bisio ${ }^{1,2}$ and Facundo García-Bournissen ${ }^{1,2}$
}

\begin{abstract}
Background: In animal research, "refinement" refers to modifications of husbandry or experimental procedures to enhance animal well-being and minimize or eliminate pain and distress. Evaluation of drug efficacy in mice models, such as those used to study Trypanosoma cruzi infection, require prolonged drug administration by the oral route (e.g. for 20 consecutive days). However, the orogastric gavage method can lead to significant discomfort, upper digestive or respiratory tract lesions, aspiration pneumonia and even accidental death. The aim of this work was to evaluate the effect of two administration methods (conventional oral gavage vs. a refined method using a disposable tip and automatic pipette) on the efficacy of benznidazole in a murine model of $T$. cruzi infection.

Results: Both administration methods led to a rapid and persistent reduction in parasitaemia. Absence of $T$. cruzi DNA (evaluated by real-time PCR) in blood, cardiac and skeletal muscle confirmed that treatment efficacy was not influenced by the administration method used.

Conclusions: The proposed refined method for long-term oral drug administration may be a suitable strategy for assessing drug efficacy in mice models of Chagas disease and can be applied to similar murine infection models to reduce animal discomfort.
\end{abstract}

Keywords: Chagas disease animal models, Chronic treatment - Oral administration, Preclinical drug research, Refinement

\section{Background}

Drug administration by the oral route is a common method to achieve systemic exposure. Substances can be delivered to the gastrointestinal tract by including them in water or food, by oropharyngeal administration of capsules, pills or fluids, or through gavage [1] requiring physical restraint by trained staff to minimize animal harm and stress.

\footnotetext{
* Correspondence: jgulin@fvet.uba.ar

'Instituto Multidisciplinario de Investigaciones en Patologías Pediátricas (IMIPP), Hospital de Niños "Dr. Ricardo Gutiérrez", CONICET- GCBA, Gallo 1330, 1425 Buenos Aires, Argentina

${ }^{2}$ Servicio de Parasitología y Enfermedad de Chagas, Hospital de Niños "Dr. Ricardo Gutiérrez". Ministerio de Salud. GCBA, Buenos Aires, Argentina
}

When a research protocol requires chronic oral drug administration, inclusion in food or water may be possible, but may increase variability and uncertainty in the ingested dose, reducing dosing accuracy. In addition, many compounds are not water-soluble, and environmental conditions such as temperature and humidity can affect stability [2].

Oral gavage delivers a known drug amount in a single administration step. Though effective, it requires substantial technical skills, is very labor-intensive and is not suitable for long-term and/or frequent treatments. Disadvantages of gavage dosing include risks of esophageal or stomach damage and inadvertent administration into the airway using both house-made and commercial devices systems [3]. 
In animal research, "refinement" refers to "modifications of husbandry or experimental procedures to enhance animal well-being and minimize or eliminate pain and distress" [4]. While many efforts have been done to refine post-surgical analgesia administration $[5,6]$ to minimize stress interference in behavior studies [7] or in neuroleptic therapy models [8] there is still a lack of information on the bias that less invasive oral administration methods may introduce in parasitology models.

Treatment options for Chagas disease rely on benznidazole (BZ) and nifurtimox (NFX), developed over four decades ago and still the only available chemotherapy, with potential severe adverse effects. Thus, there is an urgent need for new and better chemotherapy for this endemic parasitic disease [9].

In this context, a consensus for pre-clinical drug discovery for Chagas disease has emerged, which proposes a 20 consecutive day treatment protocol using the oral route to evaluate the efficacy of new anti-Trypanosoma cruzi compounds in the murine model of acute infection [10].

To alleviate potential drawbacks of the gavage method, we developed a refined method of oral administration using a disposable tip and automatic pipette. The aim of this work is to compare both oral administration methods (i.e. oral gavage vs. the refined method using a disposable tip with an automatic pipette) on the efficacy of $\mathrm{BZ}$ in a murine model of $T$. cruzi infection during a twenty-consecutive day protocol.

\section{Results}

Animal welfare

Benznidazole treatment was well tolerated by mice throughout the study whatever the administration method. Weight and body temperature were registered regularly and there were no statistical differences between both administration methods (data not shown). Mice receiving treatment through traditional oral gavage displayed aversion to the procedure and the discomfort lasted for the 10-15 min after dosage (noted as immobility, gagging and respiratory distress). Conversely, the refined method was well accepted and all mice received the full volume of the drug. Mice treated with the refined method seemed normal and resumed their usual behavior immediately after drug administration. There was no damage observed on the hard palate during or immediately after drug administration and there no substance loss during the treatment. All mice survived the treatment period in both administration methods.

\section{Treatment and assessment of cure}

Parasitaemia was evaluated daily starting on the 7th day post-infection (dpi) by direct microscopy. All infected animals displayed patent parasitaemia at $12 \mathrm{dpi}$, and BZ treatment started immediately (Fig. 1). Since ShapiroWilk tests analysis showed that parasitaemia values did not approximate to normality $(p=0.43$ for TIP $p=0.53$ for GAV), nonparametric tests were used for analysis.

Acute infection was established similarly in both groups. Although at treatment onset TIP group had

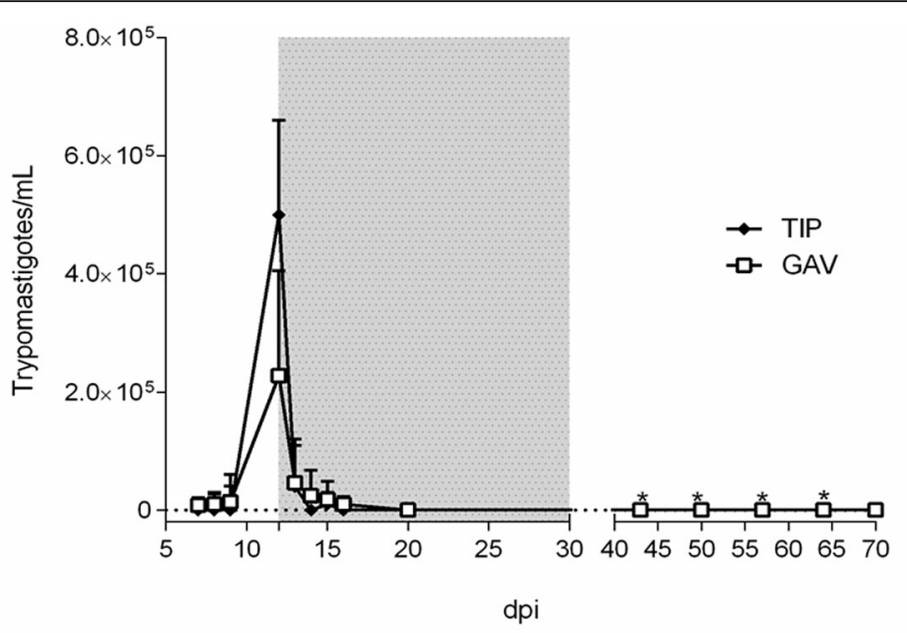

Grey shading indicates the treatment period. Asterisks $\left({ }^{*}\right)$ shows CYP administration. BALB/C mice were infected with 1,000 bloodstream trypomastigotes of $T$. cruzi VD strain. Data obtained from five mice per group are expressed as median and error bars showing standard deviations. dpi: day post infection. CYP: cyclophosphamide ( $200 \mathrm{mg} / \mathrm{kg}$, ip route).

Fig. 1 Parasitaemia curve in infected mice treated with benznidazole using gavage (GAV) or tip method (TIP) 
significantly higher mean parasitaemia values than GAV $\left(4.84 \times 10^{5}\right.$ vs. $2.28 \times 10^{5} ; p=0.03$; Mann-Whitney $)$ parasitaemia course was similar between experimental groups and as expected with the acute phase of infection by the $T$. cruzi VD strain (Fig. 1).

A median of $4 \mathrm{BZ}$ doses (range 1 to 5 doses) were needed to reach parasitaemia clearance in the TIP administration group, while and in the GAV group, a median of $5 \mathrm{BZ}$ doses were required (range 1 to 5 doses). The number of doses to achieve parasitaemia clearance were not statistically different $(p=0.59$; Mann Whitney test). At the end of treatment, (i.e., $31 \mathrm{dpi}$ ), all animals had negative parasitaemia by direct blood observation and no mortality was observed in any group.

Similarly, no differences were detected in median patent parasitaemia period between both administration methods: 4 days for TIP group (range 1 to 9 days) and 8 days for GAV group (range 1 to 8 days) $(p=0.88$; Mantel-Cox log rank sum) (Fig. 2).

Immunosuppression cycles started at $43 \mathrm{dpi}$ and were repeated at 50, 57 and $64 \mathrm{dpi}$. As no mice developed rebound parasitaemia by direct blood examination, parasitological cure was explored by qPCR in blood, heart, and skeletal muscle (Table 1). All heart samples but one (from GAV group) were negative while skeletal muscle samples were negative or below the limit of quantitation by qPCR therefore the animals were considered cured.

\section{Discussion}

This work was conducted to validate the efficacy and safety of a new, simple and low-cost method for daily oral drug administration using a murine model of $T$. cruzi acute infection. This method is potentially transferable to other rodent preclinical studies.
This refined strategy involves using a disposable tip and automatic pipette, which allows selecting final volume to the minimum required volume to dissolve the compound (e.g.: 20 or $50 \mu \mathrm{L}$ ), avoiding animal exposure to unnecessarily large volumes. Although this refined method is already in use in our protocols, we thought that it would be important to validate it in order to use it as a routine procedure for long-term oral drug administration in drug research and development.

To the best of our knowledge, up to now there were no studies evaluating compound efficacy in animal models of infectious or parasitological diseases while using a more refined oral administration method. Previous work reported morbi-mortality caused by repeated oral gavage drug administration in a murine model of $T$. cruzi infection, a situation that can potentially distort valuable results when studying new chemotherapies for Chagas disease and other diseases [11].

The refined oral administration method offers many advantages over conventional gavage administration, such as less animal handling time, less intensive operator training and, most important, reduced risk of upper respiratory and digestive tract damage or aspiration pneumonia in those protocols that require chronic oral compound administration. In addition, this method allows for accurate individual dose and volume tailoring.

A similar refined technique was described for 15 and 40 days-old rats previously trained for water deprivation with good acceptance [7]. Unlike this report, none of the animals in our study refused to drink BZ compound and there was no needed to train the animals nor submit to water deprivation period prior to dosing.

A potential disadvantage of this method is that treatment acceptance by the animals would be dependent on

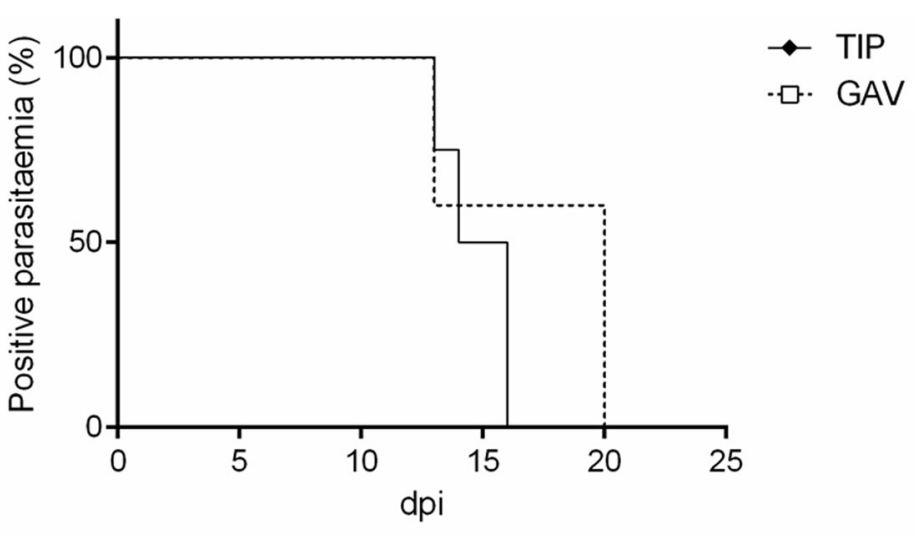

Treatment with benznidazole ( $100 \mathrm{mg} / \mathrm{kg} / \mathrm{day}$, for 20 consecutive days) started $12 \mathrm{dpi}$. Oral gavage method-treated mice (GAV), dashed broken line; Oral tip method-treated mice (TIP), continuousline.

Fig. 2 Cumulative median time to parasitaemia clearance in mice infected with Trypanosoma cruzi 
Table 1 Trypanosoma cruzi detection after immunosuppression cycle in a murine model of acute infection

\begin{tabular}{llllll}
\hline $\begin{array}{l}\text { Administration } \\
\text { method }\end{array}$ & $\begin{array}{l}\text { Number } \\
\text { of mice } \\
\text { with } \\
\text { parasite } \\
\text { rebound } \\
\text { FBE (\%) }\end{array}$ & Blood & Skeletal muscle & Heart & $\begin{array}{l}\text { Mice with } \\
\text { parasitological } \\
\text { cure (\%) }\end{array}$ \\
\hline GAV & $0 / 5(0 \%)$ & $0 / 5(0 \%)$ & $\begin{array}{l}0 / 5(0 \%) \\
\left(4^{\mathrm{a}}\right)\end{array}$ & $\begin{array}{l}1 / 5(20 \%) \\
\left(1^{\mathrm{a}}\right)\end{array}$ & $5 / 5(100 \%)$ \\
TIP & $0 / 5(0 \%)$ & $0 / 5(0 \%)$ & $0 / 5(0 \%)$ & $0 / 5(0 \%)$ & $5 / 5(100 \%)$ \\
\hline
\end{tabular}

FBE fresh blood examination, GAV oral administration method by gavage, TIP oral administration method by pipette and tip

${ }^{a}$ Number of samples with $T$. cruzi DNA detectable but below the quantization limit $(\mathrm{Ct}>30)$

drug palatability, which may add variability to the efficacy depending on the taste of the drug and physicochemical characteristics of the administered solution with transient discomfort and a higher risk of substance loss. Although this situation was no observed in our work, palatability is a factor to consider prior to establishing a more refined technique procedure [12].

This acute model of $T$. cruzi infection was previously standardized and the employed VD strain shows high parasitaemia levels and high mortality rates in non-treated (NT) infected BALB/c mice [13]. Then, for this work, there was no NT group added since the aim was to present an alternative oral administration method with a reference drug. BZ was chosen for our study based on the proven efficacy and current use in experimental models and clinical cases of Chagas disease [10, 14].

BZ effectiveness (i.e. parasitological cure) was established both by the absence of rebound parasitaemia after immunosuppression and quantifiable parasite DNA detection in blood and target tissues assessed by qPCR. The absence of parasitaemia rebound was confirmed by qPCR and all blood samples were negative irrespective of the administration method.

Although T. cruzi DNA was detected in some samples from cardiac and skeletal muscle from both groups, the obtained signal was below the quantitation limit. It is important to note that VD strain shows a high tropism for skeletal muscle and therefore many $T$. cruzi DNA may be present at the site of infection [13]. Persistence of parasite DNA detectable but not quantifiable was consistent with parasitological cure in our model, as these results can be explained by the persistence of $T$. cruzi DNA in local phagocytes (as documented in previous studies [15] that may represent intra and/or extracellular granular antigens, abundant in heart and skeletal muscle during the acute phase of the infection [16].

CYP is a commonly used agent to establish immunodepletion in mice [10]. Two CYP injections at $150 \mathrm{mg} / \mathrm{kg}$ by intraperitoneal route are enough to produce severe leukopaenia [17]. Thereby, the CYP protocol used in our work can be considered enough to lead to parasitaemia rebound if there were any persisting parasites in sanctuary sites, such as heart and skeletal muscle due to BZ failure.

Previous studies in other experimental models have yielded similar observations. In a murine Echinococcus multilocularis experimental model, albendazole serum concentrations were similar when administered either by gavage or by voluntary ingestion in honey mixture, and a comparable antiparasitic activity achieved [2].

Although no pharmacokinetic analyses have been done yet, our results suggest than BZ efficacy is not affected by either administration method, and given the rapid absorption of oral BZ into circulation it is unlikely that significant pharmacokinetics differences would exist among both methods [18].

Due to the lack of similar studies, there was no previous data available on which to base an a priori power analysis. Moreover, our experimental outcome was a categorical variable (cured/no cured dichotomy), hampering sample size estimation beforehand. Accordingly, we assumed that the refined administration method should be as effective as the standard. Considering a complete treatment response in mice treated with $\mathrm{BZ}$ by gavage method (gold standard), it would be unacceptable to obtain biased results due to the administration method apart from the drug activity.

\section{Conclusions}

As no significant differences were detected in drug efficacy between both administration methods, this refined technique consisting on administration with a tip and automatic pipette seems like a reliable method for BZ daily oral administration in an acute experimental murine model of $T$. cruzi infection while not affecting the experimental outcome and it could even be applied to similar murine models of related infectious or parasitic diseases.

\section{Material and methods}

\section{Animals}

Twenty-one days-old BALB/cJ male mice obtained from Animal Facilities (Faculty of Veterinary Sciences, 
University of Buenos Aires) were housed under conventional closed barriers at Dr. Ricardo Gutiérrez Buenos Aires Children's Hospital animal facilities. Animals were acclimatized to new housing conditions and habituated to routine handling by trained personnel for 7 days prior to the experiment.

Mice were individually identified and housed in properly labelled, $600 \mathrm{~cm}^{2}$ polycarbonate cages at 5 mice per cage. Cages were filled with irradiated chip-bedding and changed once a week. Mice had ad libitum access to food (Rata-Ratón Cooperación ${ }^{\circ}$, Argentina) and water filtered by reverse osmosis. Macroenvironmental conditions included 12:12-h light: dark cycle (starting at 6 a.m.), a controlled temperature in a range of 20 to $22^{\circ} \mathrm{C}$, and humidity from 45 to $55 \%$.

The study protocol was approved by the Institutional Animal Care and Use Committee from the Faculty of Veterinary Sciences - University of Buenos Aires (\# Protocol: 2014/4).

\section{Experimental infection}

Mice (28-35 days old; $20.12 \pm 2.35$ g) were infected with 1000 bloodstream trypomastigotes of T. cruzi VD strain, previously characterized [13] and maintained by serial passages in outbred CF-1 male mice. The inoculum was suspended in sterile RPMI-1640 medium and administered in a final volume of $0.2 \mathrm{~mL}$ per animal by intraperitoneal (ip) route. Treatment started at 12 days post infection (dpi), when all infected animals exhibited bloodstream trypomastigotes and it was randomly assigned as follows: GAV: BZ administered by gavage $(n=5)$; or TIP: BZ administered by a disposable tip and automatic pipette $(n=5)$.

\section{Drug and treatment administration}

BZ commercial tablets (Radanil ${ }^{\circ}$, Roche) were crushed and suspended in a $0.25 \%$ solution of sodium carboxymethyl cellulose (Sigma-Aldrich Co.). Treatment was administered orally at a dose of $100 \mathrm{mg} / \mathrm{kg} /$ day for 20 consecutive days, between 10 and 11 a.m. All animals were weighed at least once a week, and drug doses adjusted for weights. Dose, length of treatment and route of administration were chosen based on published data [10].

For gavage dosing, a disposable and flexible gavage with an $8-10 \mathrm{~cm}$ long $35 \mathrm{~K}$ nasogastric tube was prepared. This device was then coupled to a $1 \mathrm{~mL}$ syringe. The oral gavage technique was carried out as previously described [1] (Fig. 3a).

Pipette tip administration was performed with disposable tips $\left(\mathrm{Kima}^{\circ}\right.$, Italy, \#18170) and a $200 \mu \mathrm{L}$ volume automatic pipette. This refined administration technique consisted in filling the pipette with the drug solution at the corresponding volume, along with physical restraining of the mouse with the least skilful hand and the pipette in the other hand. Then, the tip was slightly approached to the animal's mouth and the volume was discharged slowly into the oral cavity, while gently pressing the hard palate with the tip to stimulate the swallowing reflex (Fig. 3b). The procedure was usually fast, and restraint was limited to less than $30 \mathrm{~s}$ since the volume to be dispensed was generally low $(50-100 \mu \mathrm{L})$.

\section{Assessment of treatment response}

Parasitaemia was evaluated since 7 dpi every day until it became negative for at least three consecutive days, using a modified Pizzi-Brener method [19-21]. A total of $5 \mu \mathrm{L}$ of blood was taken by amputation of the tail tip.
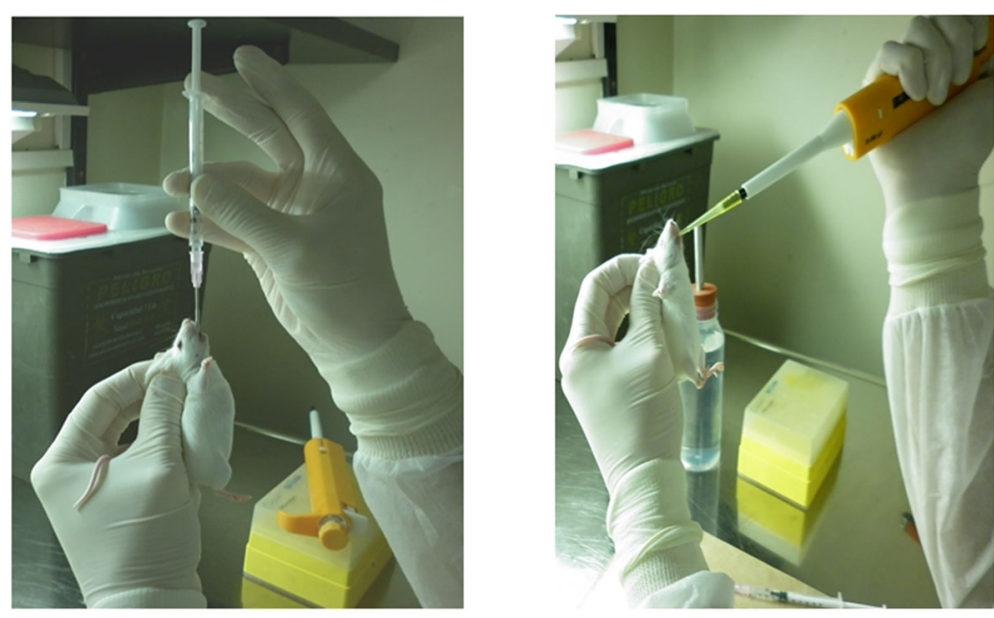

(A) Picture showing gavage method. (B) Picture showing the refined administration method with tip and automated pipette.

Fig. 3 Mice receiving oral dosing of benznidazole 
In the successive sampling, the clot was removed and the blood was extracted by milking the tail gently [22].

Then, the sample was compressed between a glass slide and an $18 \times 18 \mathrm{~mm}$ coverslip and examined microscopically at $\times 400$ magnification. The number of parasites per $\mathrm{mL}$ of blood was determined by counting trypomastigotes in 50 fields and then multiplying that number by a conversion factor $\left(10^{4}\right)$ to express the result in terms of trypomastigotes $/ \mathrm{mL}$. This technique allows detecting a minimum of 10,000 trypomastigotes $/ \mathrm{mL}$. Patent period (defined as the time elapsed between parasitaemia onset and parasitaemia clearance) and the number of doses to obtain parasite clearance were also recorded.

After treatment was completed, animals with undetectable parasitaemia were left without treatment for 10 days, with regular blood testing for re-emergence of bloodstream parasites, and then subjected to a cyclophosphamide (CYP)-based immunosuppression protocol to rule out parasite recurrence from sanctuary sites in tissues. Immunosuppression protocol consisted of 4 cyclophosphamide administrations (CYP) (Filaxis Laboratory, Buenos Aires, Argentina), at $200 \mathrm{mg} / \mathrm{kg}$ by ip route, separated by 7 days [23].

Parasitaemia was evaluated weekly during immunosuppression; finally, animals that remained negative were euthanized with sodium thiopental (Hipnopento ${ }^{\circ}$, Dr. Gray Products, Argentina) at $300 \mathrm{mg} / \mathrm{kg}$ by ip route. At the moment of euthanasia blood, skeletal and heart muscle were collected for T. cruzi-DNA testing by realtime PCR (qPCR).

\section{Preparation of peripheral blood and tissues for qPCR}

One hundred microlitres of blood were obtained by submandibular vein puncture [24], collected in a sterile tube and mixed immediately with $200 \mu \mathrm{L}$ of guanidine $6 \mathrm{M}$ EDTA $0.2 \mathrm{M}$ buffer. Rear legs skeletal and heart muscle samples $(25-50 \mathrm{mg})$ were obtained with sterile scissors and were rinsed with sterile distilled water before collection into separated DNase-free tubes. Samples were stored at $-20^{\circ} \mathrm{C}$ until processing.

\section{DNA preparation and q-PCR}

Total genomic DNA was extracted from blood and tissues using the High Pure PCR Template Preparation Kit (Roche ${ }^{\circ}$. T. cruzi DNA amplification was performed on a StepOne PCR system (Applied Biosystems ${ }^{\circ}$ ) as described elsewhere [25]. Amplification product detection was performed using TaqMan ${ }^{\circ}$ probe cruzi 3 labelled with 5'FAM (6 - carboxyfluorescein) and 3'MGB (minor groove binder).

Each 48-well reaction plate contained two positive and two negative controls. Negative controls consisted of a reaction with $T$. cruzi-specific primers without DNA and with DNA extracted from blood or tissues from noninfected mice. PCR standard curves were generated by inoculating specimens with $8 \times 10^{5}$ trypomastigotes of $T$. cruzi VD strain, followed by DNA extraction and serial dilutions. The standard curve allowed DNA quantification in the range of $1.6 \times 10^{0}$ to $8 \times 10^{5}$ parasite equivalents $/ \mathrm{mL}$. Amplification efficiencies were determined by StepOne Software v2.1 (Applied Biosystems ${ }^{\circ}$ ) by calculating efficiency $(E)=10^{(-1 / \text { slope })}$. Parasite loads in individual specimens were calculated based on the standard curve included in each batch run.

\section{Experimental design and statistical analysis}

No previous information was available to estimates the sample size and power analysis for this pilot study. Thus, the sample size was based on published suggestions to assess the efficacy of new compounds in mice models of $T$. cruzi infection [26]. Male mice were chosen since they are known to be more susceptible to $T$. cruzi infection than females, which would maximize the chances to observe a difference in response between interventions [27].

Each experimental group was composed of 4- and 5weeks old mice at the moment of infection, and each cage was occupied by animals from different experimental groups, unequivocally identified.

Nonparametric tests were used when graphical inspection of data Shapiro-Wilk test indicated that data did not approximate to normal distribution and could not be transformed to meet assumptions for parametric analyses.

Non-parametric Mann-Whitney test was performed to assess differences in maximum parasitaemia values, and number of doses needed to achieve parasitaemia clearance between both administration methods, while differences in patent parasitaemia was analyzed with Mantel-Cox rank test. The statistical significance level was determined a priori at a $P$-value $<0.05$. Values are expressed as median and range. All analysis and graphics were performed with GraphPad Prism (GraphPad Prism, Version 5.03 for Windows, Graph- Pad Software, San Diego, CA, USA; www.graphpad.com).

\section{Acknowledgments}

We thank Dr. María Elisa Solana (Instituto de Investigaciones en Microbiología y Parasitología Médica (IMPAM). UBA-CONICET, Buenos Aires, Argentina) for kindly providing VD strain of T. cruzi.

\section{Authors' contributions}

FGB and JENG have contributed significantly by designing the experiments. JENG and MB have contributed significantly by conducting the experiments and collecting the data. All authors have analyzed the data and writing the manuscript. All authors read and approved the final manuscript.

\section{Authors' information}

JENG is a Doctoral Fellow from Consejo Nacional de Investigaciones Cientificas y Técnicas (CONICET), Argentina. MB and FGB are members of the Research Career Programme from CONICET. 


\section{Funding}

The authors received no funding for this work.

\section{Availability of data and materials}

The datasets during and/or analyzed during the current study available from the corresponding author on reasonable request.

\section{Ethics approval}

The study protocol was approved by the Institutional Animal Care and Use Committee from the Faculty of Veterinary Sciences - University of Buenos Aires (\# Protocol: 2014/4).

\section{Consent for publication}

Not applicable.

\section{Competing interests}

The authors declare that they have no competing interests.

Received: 26 June 2020 Accepted: 11 October 2020

Published online: 20 October 2020

\section{References}

1. Morton D, Jennings M, Buckwell A, Ewbank R, Godfrey C, Holgate B, et al. Refining procedures for the administration of substances. Report of the BVAAWF/FRAME/RSPCA/UFAW Joint Working Group on Refinement. British Veterinary Association Animal Welfare Foundation/Fund for the Replacement of Animals in Medical Experiments/Royal so. Lab Anim. 2001; 35(1):1-41.

2. Küster T, Zumkehr B, Hermann C, Theurillat R, Thormann W, Gottstein B, et al. Voluntary ingestion of antiparasitic drugs emulsified in honey represents an alternative to gavage in mice. J Am Assoc Lab Anim Sci. 2012; 51(2):219-23.

3. Zimmer J, Lewis S, Moyer J. Comparison of gavage, water bottle, and a high-moisture diet bolus as dosing methods for quantitative Dxylose administration to B6D2F1 (Mus musculus) mice. Lab Anim. 1993;27(2):164-70.

4. National Research Council. Guide for the care and use of laboratory animals. 8th. National Research Council, editor. Washington, D.C.: The National Academic Press; 2011. 248.

5. Abelson KSP, Jacobsen KR, Sundbom R, Kalliokoski O, Hau J. Voluntary ingestion of nut paste for administration of buprenorphine in rats and mice. Lab Anim. 2012;46(4):349-51.

6. Kalliokoski O, Jacobsen KR, Hau J, Abelson KSP. Serum concentrations of buprenorphine after oral and parenteral administration in male mice. Vet J. 2011;187(2):251-4.

7. Wheeler TL, Eppolito AK, Smith LN, Huff TB, Smith RF. A novel method for oral stimulant administration in the neonate rat and similar species. J Neurosci Methods. 2007:159(2):282-5.

8. Schleimer $\mathrm{S}$, Johnston $\mathrm{G}$, Henderson J. Novel oral drug administration in an animal model of neuroleptic therapy. J Neurosci Meth. 2005;2:159-64.

9. Chatelain E. Chagas disease research and development: is there light at the end of the tunnel? Comput Struct Biotechnol J. 2017;15:98-103.

10. Romanha A, Castro S, Soeiro MN, Lannes-Vieira J, Ribeiro I, Talvani A, et al. In vitro and in vivo experimental models for drug screening and development for Chagas disease. Mem Inst Oswaldo Cruz. 2010;105(2):233-8.

11. Buckner F, Bahia M, Suryadevara P, White K, Shackleford D, Chennamaneni $\mathrm{N}$, et al. Pharmacological characterization, structural studies, and in vivo activities of anti-Chagas disease lead compounds derived from tipifarnib. Antimicrob Agents Chemother. 2012;56(9):4914-21.

12. Atcha Z, Rourke C, Neo A, Goh C, Lim J, Aw C, et al. Alternative method of oral dosing for rats. J Am Assoc Lab Anim Sci. 2010;49(3):335-43.

13. Gulin J, Bisio M, Rocco D, Altcheh J, Solana M, García-Bournissen F. Molecular and biological characterization of a highly pathogenic Trypanosoma cruzi strain isolated from a patient with congenital infection. Exp Parasitol. 2018;186:50-8.

14. Bern C. Chagas' disease. N Engl J Med. 2015;373(5):456-66.

15. Martins H, Figueiredo L, Valamiel-Silva J, Carneiro C, Machado-Coelho G, Vitelli-Avelar D, et al. Persistence of PCR-positive tissue in benznidazoletreated mice with negative blood parasitological and serological tests in dual infections with Trypanosoma cruzi stocks from different genotypes. J Antimicrob Chemother. 2008;61:1319-27.
16. Guarner J, Bartlett J, Zaki S, Colley D, Grijalva M, Powell M. Mouse model for Chagas disease: immunohistochemical distribution of different stages of Trypanosoma cruzi in tissues throughout infection. Am J Trop Med Hyg. 2001;65(2):152-8.

17. Huyan $X$, Lin Y, Gao T, Chen R, Fan Y. Immunosuppressive effect of cyclophosphamide on white blood cells and lymphocyte subpopulations from peripheral blood of BALB/c mice. Int Immunopharmacol. 2011;11(9):1293-7.

18. Perin L, Da Silva RM, Da Silva FK, De Oliveira Cardoso JM, Mathias FAS, Reis LES, et al. Pharmacokinetics and tissue distribution of benznidazole after oral administration in mice. Antimicrob Agents Chemother. 2017;61(4):1-30.

19. Brener Z. Therapeutic activity and criterion of cure on mice experimentally infected with Trypanosoma cruzi. Rev Inst Med Trop Sao Paulo. 1962;4(6):389-96.

20. Gulin J, Eagleson M, Postan M, Cutrullis R, Freilij H, García-Bournissen F, et al. Efficacy of voriconazole in a murine model of acute Trypanosoma cruzi infection. J Antimicrob Chemother. 2013;68(4):888-94.

21. Simões-Silva MR, De Araújo JS, Oliveira GM, Demarque KC, Peres RB, D'Almeida-Melo I, et al. Drug repurposing strategy against Trypanosoma cruzi infection: in vitro and in vivo assessment of the activity of metronidazole in mono- and combined therapy. Biochem Pharmacol. 2017;145:46-53.

22. Christensen S, Mikkelsen L, Fels J, Bodvarsdóttir T, Hansen A. Quality of plasma sampled by different methods for multiple blood sampling in mice. Lab Anim. 2009:43(1):65-71.

23. Antoine-Moussiaux N, Saerens D, Desmecht D. Flow cytometric enumeration of parasitaemia and haematologic changes in trypanosomainfected mice. Acta Trop. 2008;107:139-44.

24. Golde W, Gollobin P, Rodriguez L. A rapid, simple, and humane method for submandibular bleeding of mice using a lancet. Lab Anim (NY). 2005;34(9):39-43.

25. Piron M, Fisa R, Casamitjana N, López-Chejade P, Puig L, Vergés M, et al. Development of a real-time PCR assay for Trypanosoma cruzi detection in blood samples. Acta Trop. 2007 Sep;103(3):195-200.

26. Croft S, Brun R. In vitro and in vivo models for the identification and evaluation of drugs active against Trypanosoma and Leishmania. In: Drugs against parasitic diseases: R\&D methodologies and issues discoveries and drug development. 1st ed. Geneva: TDR, WHO; 2003.

27. De Souza EM, Rivera M, Araújo-Jorge TC, De Castro SL. Modulation induced by estradiol in the acute phase of Trypanosoma cruzi infection in mice. Parasitol Res. 2001;87:513-20.

\section{Publisher's Note}

Springer Nature remains neutral with regard to jurisdictional claims in published maps and institutional affiliations.

Ready to submit your research? Choose BMC and benefit from:

- fast, convenient online submission

- thorough peer review by experienced researchers in your field

- rapid publication on acceptance

- support for research data, including large and complex data types

- gold Open Access which fosters wider collaboration and increased citations

- maximum visibility for your research: over $100 \mathrm{M}$ website views per year

At BMC, research is always in progress.

Learn more biomedcentral.com/submissions 